\title{
The impact of the SARS-CoV-2 pandemic and COVID-19 on lung transplantation in the UK: Lessons learned from the first wave
}

\section{Gillian Hardman $^{1,2}$ (c) | Ruth Sutcliffe ${ }^{3,4}$ | Rachel Hogg ${ }^{5}$ | Lisa Mumford ${ }^{5}$ Laura Grocott $^{6}$ | Sarah-Jane Mead-Regan ${ }^{7}$ | Jane Nuttall ${ }^{4}$ | Stephanie Dunn ${ }^{8}$ | Philip Seeley $^{9}$ | Stephen Clark $^{2}$ | Richard Quigley ${ }^{10}$ | Nawwar Al-Attar ${ }^{11}$ | Karen Booth ${ }^{2}$ | John H. Dark ${ }^{12}$ | Andrew J. Fisher ${ }^{2,12}$ | the NHS Blood, Transplant Cardiothoracic Advisory Group Clinical Audit Group}

\footnotetext{
${ }^{1}$ Clinical Research and Clinical Audit Fellow in Cardiothoracic Transplantation, NHS Blood and Transplant, Bristol, UK

${ }^{2}$ Institute of Transplantation, Freeman Hospital, Newcastle upon Tyne, UK

${ }^{3}$ Allied Health Professional representative, Cardiothoracic Advisory Group Clinical Audit Group, NHS Blood and Transplant, Bristol, UK

${ }^{4}$ Recipient Transplant Coordinator, Wythenshawe Hospital, Manchester, UK

${ }^{5}$ Statistics and Clinical Studies, NHS Blood and Transplant, Bristol, UK

${ }^{6} \mathrm{Heart}$ and Lung transplant recipient coordinator, Queen Elizabeth Hospital, Birmingham, UK

${ }^{7}$ Heart and Lung Transplant Recipient Coordinator, Great Ormond Street Hospital for Children, London, UK

${ }^{8} \mathrm{Heart}$ and Lung transplant recipient coordinator, Institute of Transplantation, Freeman Hospital, Newcastle upon Tyne, UK

${ }^{9}$ Lead Nurse for Heart and Lung Transplantation, Freeman Hospital, Newcastle upon Tyne, UK

${ }^{10}$ Lead Nurse for transplantation, Royal Papworth Hospital, Cambridge, UK

${ }^{11}$ Chair, Cardiothoracic Advisory Group Clinical Audit Group, NHS Blood and Transplant, Bristol, UK

${ }^{12}$ Newcastle University Translational and Clinical Research Institute, Newcastle upon Tyne, UK
}

\section{Correspondence}

Gillian Hardman, Institute of

Transplantation, Freeman Hospital,

Freeman Road, High Heaton, Newcastle

upon Tyne NE7 7DN, UK.

Email: Gillian.hardman@nhsbt.nhs.uk

\begin{abstract}
Background: Lung transplantation is particularly susceptible to the impact of the severe acute respiratory syndrome coronavirus-2 (SARS-CoV-2) pandemic, and evaluation of changes to practice is required to inform future decision-making.

Methods: A retrospective review of the UK Transplant Registry (UKTR) and national survey of UK lung transplant centers has been performed.

Results: There was geographic variation in the prevalence of COVID-19 infection across the UK. The number of donors fell by $48 \%$ during the early pandemic period. Lung utilization fell to $10 \%$ (compared with $24 \%$ for the same period of 2019). The number of lung transplants performed fell by 77\% from 53, March to May 2019, to 12. Seven (58\%) of these were performed in a single-center, designated "COVID-light." The number of patients who died on the lung transplant waiting list increased, compared to the same period of 2019 ( $p=.0118$ ). Twenty-six lung transplant recipients with confirmed COVID-19 infection were reported during the study period.
\end{abstract}


Conclusion: As the pandemic continues, reviewing practice and implementing the lessons learned during this period, including the use of robust donor testing strategies and the provision of "COVID-light" hospitals, are vital in ensuring the safe continuation of our lung transplant program.

\section{KEYWORDS}

coronavirus pandemic, COVID-19, lung transplantation, organ donation, SARS-CoV-2

\section{1 | INTRODUCTION}

Severe acute respiratory syndrome coronavirus-2 (SARS-CoV-2), the causative agent for coronavirus disease-2019 (COVID-19), was first diagnosed in the United Kingdom (UK) in January $2020 .{ }^{1}$ With in creasing case numbers, unprecedent public health measures aimed at containing the spread of the virus and mitigating the impact of a surge of cases on the National Health Service (NHS), were undertaken, including a national lockdown on March 23, 2020. ${ }^{2}$ After the number of COVID-19 cases fell during May/June, initial lockdown measures were eased. However, new restrictions are being increasingly implemented at the time of writing.

For transplant programs, hospital resource requirements, the availability and safety of deceased organ donors and the safety of transplant candidates and recipients, have forced difficult decisions and changes to practice, with early reports from across the world identifying significant decreases in solid organ transplantation, ${ }^{3-7}$ including lung transplantation ${ }^{8,9}$ programs, in response to the pandemic.

As a novel disease, and unprecedented global pandemic, the evidence base informing practice was initially limited, albeit growing rapidly throughout the course of the pandemic. During the early phase in the UK, guidance for solid organ donation was issued at a national level, from National Health Service Blood and Transplant (NHSBT), with individual organ programs, and transplant centers, making decisions locally, based on the needs of their transplant patient population, balancing the risks of COVID-19 infection, and the prevalence of the disease locally, and its subsequent impact on hospital resource.

As the pandemic continues, evaluation of national and regional changes to lung transplant practice in the UK, and their impact on the patient population, during the early phase of the pandemic, is required, to inform future decision-making. The aim of this work was to understand changes to clinical practice during the early pandemic period and the impact of these changes on the UK lung transplant program.

\section{2 | METHODS}

\subsection{Study population}

All adult and paediatric lung transplant centers in the UK were included in the study. Analysis included all adult (aged 16 years and older) and pediatric organ donors, lung transplant waiting list candidates, and lung transplant recipients. Heart-lung candidates and recipients were not included in the analysis.

\subsection{Study design}

A retrospective review of data submitted to the UK Transplant Registry (UKTR) from January 1, 2019, to June 30, 2020, was performed. The early pandemic period is defined here as March 1 to May 31, 2020. Month-to-month and center-level variation in practice was reviewed from January 1 to June 30, 2020, to include the early pandemic period, and, where appropriate, compared to data from January 1 to June 30, 2019.

Eligible, potential lung donors are defined as those aged less than 70 years, with no absolute contraindications to organ donation. Lung utilization was calculated as number of donors transplanted from the number of donors (who donated at least 1 solid organ) from whom the lung(s) were offered for transplantation. Analysis included lung transplant activity, early (30-day) mortality for recipients transplanted during the pandemic period, lung transplant candidate listing, and waiting list activity, including mortality and COVID-19 infection in lung transplant recipients. Prospective reporting to the UKTR, of all transplant recipients, or patients on the organ transplant waiting list, with confirmed COVID-19 infection, was commenced on March 17, 2020.

To explore regional, center-level variation in clinical practice and policy, during the pandemic, a national survey of UK transplant centers was performed.

\subsection{Survey design and conduct}

The survey was developed using an iterative process based on literature review and UK organ donation and transplantation guidance issued by NHSBT and the British Transplant Society, to identify changes in lung transplantation clinical practice, including candidate assessment, recipient management, organ donation, and activity, perceptions of the prevalence of COVID-19 locally and the impact on the transplant workforce and hospital resource. The final survey was approved by members of the NHSBT Cardiothoracic Advisory Group Clinical Audit Group (CTAG CAG). The NHS England coronavirus specialty guide cardiothoracic 
escalation framework ${ }^{10}$ was used to define phases in response to the pandemic.

The survey was conducted between May 15 and June 18, 2020. At each center, a cardiothoracic transplant clinical lead and transplant coordinator were identified to complete the survey. Participants were emailed a copy of the survey. Information required to complete the survey was gathered by the nominated persons, from members of the wider team. Additional telephone interview follow-up was performed between June 20 and July 13, 2020, where further information was required, or to clarify specific survey responses.

\section{4 | Statistical analysis}

Categorical variables are expressed as absolute numbers and their relative frequencies, with percentage change compared with previous year, where appropriate. Continuous variables are expressed as mean \pm standard deviation (SD) if normally distributed, or as median and inter-quartile range (IQR) if non-normally distributed. Chi-square test and Fisher's exact test were used for comparison between groups of categorical variables. Continuous variables were compared using Student's $t$ test or Mann-Whitney $U$ test, where appropriate. Statistical significance was considered for $p<.05$. Missing values were excluded from $p$-value calculation. All statistical analysis was performed using SAS version 9.4 (SAS Institute Inc., Cary, NC, USA.)

\section{5 | Ethical approval}

Data analyses were performed by NHSBT, who maintain the UK Transplant Registry on behalf of UK transplant centers, under the UK General Data Protection Regulation (GDPR), allowing NHSBT to use patient identifiable information for service evaluation, without additional patient consent. Survey response was voluntary, with appropriate information provided to inform consent to participation.

\section{3 | RESULTS}

The survey return rate was $100 \%$. Additional information and clarification of responses were performed by telephone interview with 3 of the 6 UK lung transplant centers.

There was geographic variation in the prevalence of COVID-19 infection across the UK noted in the survey responses. The impact of the pandemic in each center varied as a result of local prevalence of the virus, resultant hospital and ICU admission, designation as an ECMO (extra-corporeal membrane oxygenation) center (2 centers) and designation of the center as "COVID-light" (1 center). Movement of each center through the pandemic phases is shown in Table 1. At the time of the survey, all centers considered themselves to be moving into the recovery phase; however, a date for return to "normal" (pre-pandemic) service had not been identified in any center. All centers recognized that changes to clinical practice, adopted during the pandemic, would persist.

\subsection{Deceased organ donation and lung utilization}

The number of deceased donors, who donated at least 1 solid organ, where the lung(s) were offered for transplantation, fell by $48 \%$, to 123 from 238 in March to May 2019. Donors after brain Death (DBD) fell from 163 to 98 (40\% decrease) and Donors after Circulatory Death (DCD) fell from 75 to 25 (67\% decrease) (Table 2). There was a significant difference in the proportion of donors by type (DCD versus DBD), during the pandemic period, compared with the same period of 2019, with DCD donors representing 32\% of the offered donor population in 2019 and $20 \%$ in 2020 ( $p=.0334)$. The characteristics of the donors, from whom the lung(s) were offered, compared with

TAB LE 1 Movement of UK lung transplant centers through pandemic phases, during the early pandemic period March to May 2020. Local and hospital virus prevalence as assessed by individual centers and pandemic phases defined by NHS England ${ }^{10}$

\begin{tabular}{|c|c|c|c|c|c|c|}
\hline & Preparation & Escalation & $\begin{array}{l}\text { Crisis } \\
\text { compensated }\end{array}$ & $\begin{array}{l}\text { Crisis } \\
\text { uncompensated }\end{array}$ & Resolution & Recovery \\
\hline $\begin{array}{l}\text { Center A } \\
\text { High local prevalence }\end{array}$ & Early March & Mid-March & Mid-March & Late March & Mid-May & In progress \\
\hline $\begin{array}{l}\text { Center B } \\
\text { High local prevalence } \\
\text { "COVID-light" designation }\end{array}$ & Early March & Not applicable & Not applicable & Not applicable & Not applicable & Not applicable \\
\hline $\begin{array}{l}\text { Center } \mathrm{C} \\
\text { ECMO center }\end{array}$ & Early March & Mid-March & Mid-March & Late March & Early May & In progress \\
\hline $\begin{array}{l}\text { Center D } \\
\text { High local prevalence } \\
\text { Moderate hospital prevalence }\end{array}$ & Early March & Mid-March & Late March & Not applicable & Early May & Late May \\
\hline $\begin{array}{l}\text { Center } \mathrm{E} \\
\text { ECMO center }\end{array}$ & Early March & Late March & EarlyApril & Mid-April & Mid-May & Late May \\
\hline Center F & February & Early March & Mid-March & Not applicable & Mid-May & Late May \\
\hline
\end{tabular}


TABLE 2 The number of deceased organ donors, who donated at least 1 solid organ, where the lungs were offered for transplantation, during the early pandemic period, compared with 2019.

\begin{tabular}{|c|c|c|c|c|c|c|c|}
\hline \multirow[b]{2}{*}{ March } & \multicolumn{2}{|c|}{$\begin{array}{l}\text { Donors after } \\
\text { brain death } \\
\text { (DBD) where } \\
\text { lung (s) were } \\
\text { offered } \\
\text { March } 1 \\
\text { to May } 31 \text {, } \\
2020 \text { (19) }\end{array}$} & \multicolumn{2}{|c|}{$\begin{array}{l}\text { Donors } \\
\text { after } \\
\text { circulatory } \\
\text { death } \\
\text { (DCD) } \\
\text { where lung } \\
\text { (s) were } \\
\text { offered } \\
\text { March } 1 \\
\text { to May } 31 \text {, } \\
2020 \text { (19) }\end{array}$} & \multicolumn{2}{|c|}{$\begin{array}{l}\text { Deceased } \\
\text { donors } \\
\text { where lung } \\
\text { (s) were } \\
\text { offered } \\
\text { March } 1 \\
\text { to May } 31 \text {, } \\
2020 \text { (19) }\end{array}$} & \multirow{2}{*}{$\begin{array}{l}\% \\
\text { change } \\
-34 \%\end{array}$} \\
\hline & 46 & (60) & 12 & (28) & 58 & (88) & \\
\hline April & 22 & (48) & 3 & (22) & 25 & (70) & $-64 \%$ \\
\hline May & 30 & (55) & 10 & (25) & 40 & (80) & $-50 \%$ \\
\hline Total & 98 & (163) & 25 & (75) & 123 & 238 & $-48 \%$ \\
\hline
\end{tabular}

those from the same period of 2019, are shown in Table 3. There was a significant decrease in donor age (from a median age of 52 in 2019 to 43 years during the pandemic period, $p=<.0001$ ) and a significant increase in the proportion of donors with a smoking history $(53 \%$ in 2019 to $66 \%$ during the pandemic period, $p=.0180$ ).

The lung utilization rate during the early pandemic period was 10\%, 13 donors transplanted from 123 donors (who donated at least 1 solid organ) where the lung(s) were offered. One donor was utilized outside of the UK. This compares to a utilization rate of $22 \%$, for the same period of 2019

In the survey responses, all centers described formal or informal, center-level changes to lung donor acceptance criteria during the pandemic. Changes described included consideration of ideal donors only, lower age limits for potential donors and increased caution when considering travel and ischemic times.

\section{2 | Lung transplant activity and post- transplant outcomes}

All centers reported a period of restricting lung transplantation to urgent or super-urgent, inpatient candidates only, at the start of the pandemic. Decision-making was based on local hospital guidance, limiting access to ICU for non-urgent patients, and consideration of potential risks to non-urgent lung transplant candidates. Following designation as a "COVID-light" hospital, 1 center continued to consider all lung transplant candidates from early April onwards. The remaining centers adopted center-level policy to consider non-urgent, ultra-low, and low-surgical risk candidates from late-April 2020, where appropriate, balancing resource capacity.

Twelve bilateral lung transplants (utilizing 12 donors) were performed in the UK during the early pandemic period. This compares to 53 lung transplants (48 bilateral and 5 single) performed for the same period of 2019 , a reduction in activity of $77 \%$. Monthly trends in donors offered for lung transplantation and lung transplant activity are shown in Figure 1. The mean number of transplants performed, per month, in 2019 was 15, compared with 9 per month, in the first 6 months of 2020. During the early pandemic period, activity fell in all centers compared with the same period of 2019 Seven of 12 transplants (58\%) represent the activity of one center. This center was designated "COVID-light" in late March 2020. The remaining activity was divided between 3 centers. In June 2020, the number of lung transplants increased to 16 , with all UK lung transplant centers performing at least 1 lung transplant.

There were 12 adult lung recipients transplanted during the early pandemic period, 6 male and 6 female, 2 urgent and 10 non-urgent, and all bilateral lung transplants. The median (IQR) age of recipients was 53 (32-61) years. The median (IQR) donor age was 31 (23-50) years, with 11 DBD and 1 DCD transplant. The 30 -day survival is 92\% (11/12 recipients). Recipient characteristics, compared with those from the same period of 2019, are shown in Table 4.

\subsection{The lung transplant waiting list}

The number of new registrations to the lung transplant waiting list fell by $68 \%$ (Table 5 ). The number of patients on the active lung transplant waiting list at 31 May 2020 increased by 5\% to 341, compared to 326 on 31 May 2019. The outcome for candidates on the lung transplant waiting list during the early pandemic period, compared with 2019, are shown in Table 6. Death on the lung transplant waiting list during the early pandemic period increased significantly with 21 deaths compared with 12 for the same period of 2019 ( $p=.0118$ ). Confirmed COVID-19 infection was reported in two candidates on the lung transplant waiting list during the study period.

\section{4 | Recipient management}

All centers reported that active efforts had been made to reduce hospital attendance for lung transplant recipients. At the time of the survey, in all cases, these changes were planned to continue. Face-to-face follow-up has been reduced to only those within the first 6 months of transplant in one center, and those within the first 12 -months of transplant in the remaining 5 centers. All centers have implemented telephone consultation follow-up, with outpatient clinic attendance only where clinical concerns have been identified. Where outpatient attendance has continued, measures have been implemented to decrease the number of patients in attendance, to reduce the use of waiting areas and implement social distancing measures. One center reported the use of online video conferencing platforms to conduct recipient follow-up.

All centers stated that no changes were made to immunosuppression therapy in long-term lung transplant recipients, who remained well. Where COVID-19 infection was suspected or confirmed, all centers reported cessation, or dose reduction, of Calcineurin inhibitors on an individual patient basis. 
TABLE 3 Characteristics of donors ( ${ }^{*}$ who donated at least 1 solid organ) where the lung(s) were offered from March 1 to May 31, 2020, and March 1 to May 31, 2019

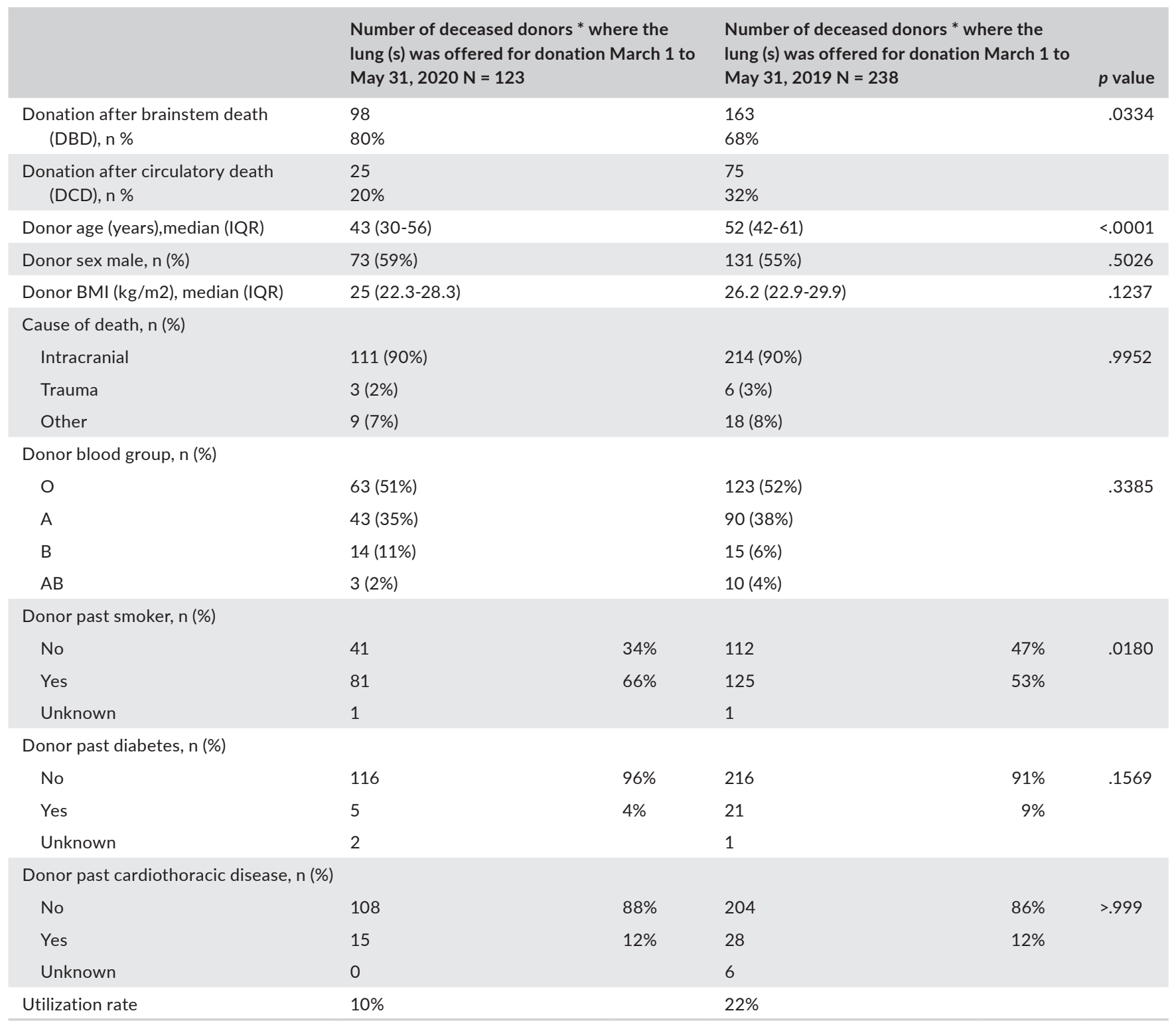

\section{5 | COVID-19 in lung transplant recipients}

Twenty-six lung transplant recipients with confirmed COVID-19 infection were reported, during the study period. Median (IQR) age of COVID-19-positive recipients is 58 years (40-65). Mean (SD) time since transplant 5.3 years (5.1). Three of the COVID-19-positive recipients had been transplanted during the early pandemic period, March to May 2020. All were listed prior to March 2020, and their indication for transplant was not related to COVID-19 infection. One recipient tested positive after hospital discharge, following recovery from their transplant. One patient had a positive result reported pre-transplantation, with a subsequent negative result, underwent lung transplant and remained asymptomatic for COVID-19. One patient had a positive pre-operative swab test, the result of which was not immediately available at the time of implantation. Due to the urgent nature, the transplant proceeded, following discussion with microbiology. All donors were negative for COVID-19 on pretransplant testing.

\section{DISCUSSION}

There was a substantial reduction in lung transplant activity in the UK during the early phase of the SARS-CoV-2 pandemic. An average of 4 lung transplants was performed each month of the early pandemic period, compared with 12.5 transplants per month during January and February of 2020. Month-to-month, and annual, variation in lung transplant activity rates is expected, and this activity compares to an average of 16 lung transplants per month, from March to May 2015 to 2019, in the UK.

The number of deceased donors from whom the lungs were offered for transplantation fell by $48 \%$, mirroring the published 


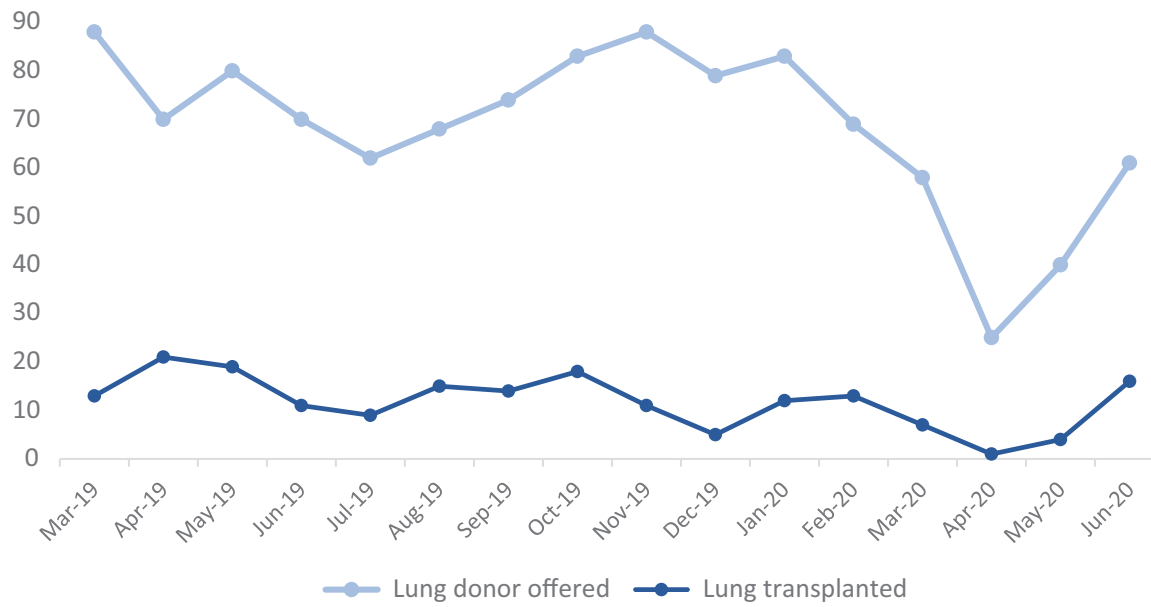

FIGURE 1 Number of deceased donors (who donated at least 1 solid organ) offered for lung transplantation and lung transplants performed, March 1, 2019, to June 30, 2020.

TAB LE 4 Recipient characteristics for lung transplants performed from March 1 to May 31, 2020, compared with March 1 to May 31 , 2019

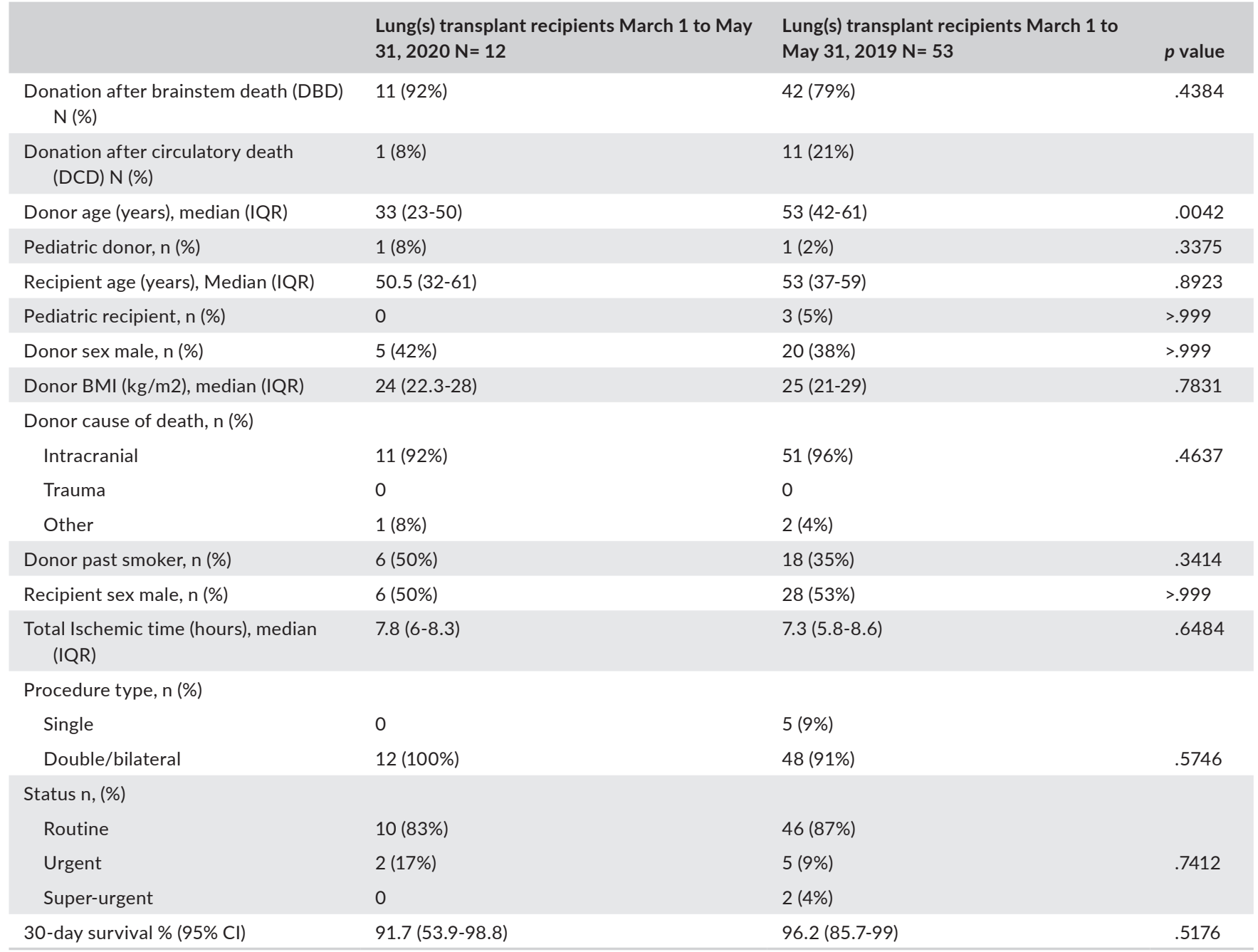

experience of other lung transplant programs, globally. ${ }^{8,9}$ Although the total number of deceased donors fell, the greatest reduction was seen in the number of donors after circulatory death (DCD), decreasing by $67 \%$. This fall occurred in response to National guidance on organ donation during this period. At the start of the early pandemic period, this national guidance placed restrictions on donor age, limiting DBD to age less than 60 years and DCD to age less than 50 years. These were gradually increased between April and June 2020. This resulted in, understandably, younger donors but also a shift in the proportions of DBD and DCD donors, with fewer DCD donors 
offered. In UK lung transplant practice over the last 5 years, DCD represents, on average, $19 \%$ of all lung transplant activity, ${ }^{11}$ a large decrease in this donor population, therefore, has the potential to significantly impact activity.

Although the number of donors from whom the lungs were offered reduced, offers for lung donation did not cease, and, as a result of national guidance for solid organ donation during this time, there were some positive changes to the donor characteristics during the study period. The median age of donors decreased significantly, from 52 years during the same period of 2019, to 43 years in 2020 . The proportion of donors with a past smoking history, however, significantly increased. This may have impacted organ acceptance and utilization, considering the center-level decision-making practices, described in survey responses, to include ideal donors only and employ a minimum risk policy, during this period.

In survey responses, staff reported confidence, which increased throughout the early pandemic period, that, where lung donor offers were received, the risk of COVID-19 infection in the donor was low and the risk of transmission to the recipient was low. This was facilitated by the development of national policy for donor screening and testing, candidate testing, and donor management practice. ${ }^{12}$ Potential donors are epidemiological screened for history of symptoms, history of positive testing or contact with a positive and/or symptomatic person. PCR testing for SARS-CoV-2 is performed in all donors using nose and throat swab as well as endotracheal aspirate. Potential recipient screening and testing are performed in the same way, using nose and throat swab samples for PCR testing. As evident from one positive case reported during the early pandemic period, transplant and organ implantation may proceed prior to a result being available, depending on clinical urgency and logistics. As the pandemic has progressed, the availability and rapidity of testing, in all donor and transplant hospitals, have improved, reducing the risk of this occurring. In line with NHSBT guidance, the use of chest CT scanning as a screening tool for COVID-19 infection has not been widely employed, and CT chest is not a routinely performed assessment in the potential lung donor population in the UK.

As the pandemic continues, and a greater number of individuals in the general population are exposed to, or will have previously tested positive for, COVID-19, new questions relating to time since exposure or positive test, and the consideration of donors with previous COVID-19 exposure, will be raised, requiring on-going synthesis of available evidence. As yet, there is no published evidence of transmission of COVID-19 from a donor to a recipient. Although the number of transplants performed from COVID-19-positive donors remains low, the first single case report in lung transplantation has been published ${ }^{13}$. In line with NHSBT guidance, donors who have previously tested positive and recovered (referring to a period of at least 28 days from full clinical recovery) may still be considered for organ donation, with liaison with microbiology/virology clinicians. As the pandemic continues, this guidance we undergo continued review.

The published experience of thoracic transplant programs around the world during this time, describe the greatest decreases in lung transplant activity focused in those areas hardest hit by the virus. ${ }^{14}$ There was similar heterogenous geographical spread of COVID-19 infection across the UK, during this early phase of the pandemic, with varying degrees of viral prevalence and subsequent hospital and intensive care unit (ICU) resource impact. It is clear, however, from individual center responses to the survey, that transplant activity was not solely affected by local prevalence of COVID-19 infection.

Here, the primary driver for the decrease in lung transplant activity appears to be a reduction in lung utilization across the UK, $10 \%$ during the early pandemic period, compared with $24 \%$ for the same period of 2019. In UK adult lung transplant practice, the utilization rate, on average, for the last 5 years, sits around 19\%. This reduction in utilization has occurred in response to local, hospital level decision-making, initially limiting solid organ transplantation, during this period, to urgent, inpatient candidates, only. Concerns about a surge in COVID-19 cases, and the subsequent demand on intensive care unit capacity, lead to restriction of access for nonurgent patients. This was in line with other national guidance for the temporary suspension of non-essential services throughout the NHS, including elective cardiothoracic surgery. For transplant clinicians, fear of COVID-19 infection in the early post-operative period, with the potential for high mortality, lead to understandable caution, when considering those candidates who were non-urgent. Urgent and super-urgent lung transplant candidates make up just 1-2\% of the lung transplant waiting list in the UK. Without available, appropriate lung transplant candidates, donors cannot be utilized and lung transplants will not be performed. Non-urgent lung transplant candidates are, as a group, distinct from other elective thoracic surgical
TABLE 5 The number of new registrations, by urgency, per month, to the lung transplant waiting list

\begin{tabular}{|c|c|c|c|c|c|c|c|c|}
\hline \multirow{2}{*}{$\begin{array}{l}\text { New registrations to the lung } \\
\text { transplant waiting list } \\
\text { March }\end{array}$} & \multicolumn{2}{|c|}{$\begin{array}{l}\text { Non- } \\
\text { urgent } \\
2020(19)\end{array}$} & \multicolumn{2}{|c|}{$\begin{array}{l}\text { Urgent } \\
2020 \\
(19)\end{array}$} & \multicolumn{2}{|c|}{$\begin{array}{l}\text { Super- } \\
\text { urgent } \\
2020 \\
(19)\end{array}$} & \multirow[t]{2}{*}{$\begin{array}{l}\text { Total } \\
2020(19)\end{array}$} & \multirow[t]{2}{*}{$\begin{array}{l}\% \\
\text { change }\end{array}$} \\
\hline & 17 & (26) & 1 & $(0)$ & 0 & (0) & & \\
\hline April & 9 & (29) & 0 & (4) & 0 & (1) & & \\
\hline May & 3 & (17) & 1 & (3) & 0 & (1) & & \\
\hline June & 4 & (21) & 0 & (4) & 0 & (3) & & \\
\hline Total & 33 & (93) & 2 & (11) & 0 & (5) & 35 (109) & $-68 \%$ \\
\hline
\end{tabular}


patients, and as such perhaps require separate classification in consideration of adoption of similar practice guidance in future.

In lung transplant practice, changes to clinical practice were driven by appropriate concerns regarding the safety of donors, hospital-acquired infection in recipients and the resource capacity required to meet the needs of COVID-19 patients. It is evident from the survey responses, that in addition to national guidance, changes to donor consideration and acceptance criteria were made, at a center-level, variably influenced by the above concerns. Allocation for non-urgent recipients in the UK is center-based, in response to group offering. Again, during the early pandemic period, lung transplant teams exhibited, understandable, increased caution when considering the risks to their candidates and this influenced decisionmaking behavior. These center-led changes to practice evolved over time during the early pandemic period and non-urgent candidates were increasingly considered. Over the early pandemic period as a whole, only 2 recipients transplanted at this time listed as urgent, the remaining 10 were non-urgent patients. Activity data from June 2020 indicate that the numbers of lung transplants are increasing, and it is hoped that this will continue, minimizing the impact of this period of reduced activity on the waiting list population. On-going analysis of UKTR data, throughout 2020 , will be required to evaluate this.

Where lung transplant activity has continued during this early pandemic period, it has been facilitated by the designation of one center as a "COVID-light" hospital. Information provided by the Royal College of Surgeons of England ${ }^{15}$ describes COVID-light as physical separation of COVID-19-positive and COVID-19-negative patients within designated areas of NHS hospitals or for an entire hospital, functioning as an NHS network hub. These areas can only be maintained with implementation of care pathways to include effective and efficient patient testing and, in future, may be facilitated by regular testing and vaccination for staff. During this period, provision of COVID-light hospitals or facilities has been determined locally, by the managing Hospital Trusts. As described here, despite high local prevalence of the virus in relation to this center, it was able to continue activity and was responsible for $58 \%$ of the lung transplants performed, during this time. In contrast, designation of some UK lung transplant centers for the provision of veno-venous ECMO (as per national NHS England specialized service commissioning), had a substantial impact on the centers' ability to continue lung transplantation. Movement of waiting list candidates to centers less affected, and continuing transplant activity, was not considered during the early pandemic period.

Detailed survival analysis of those patients transplanted during this early pandemic period has not been performed, due to the small numbers of recipients. At the time of the study, there were 3 confirmed COVID-19-positive recipients, transplanted during the pandemic period. All 3 patients were listed for transplantation prior to January 2020, for primary lung disease not related to COVID-19 infection. In each case, there is no indication that infection was transmitted from the donor to the recipient and clinical details support community-acquired COVID-19 infection.

Lung transplant waiting list candidates are particularly susceptible to the effects of this pandemic. Reduced transplant activity will result in longer waiting times and an increased risk of mortality on the waiting list. To assess the full impact on this patient population, further analysis will be required, as the pandemic progresses. Lung transplant candidates, along with lung transplant recipients, are also at increased risk of acquiring COVID-19, and developing the more severe, life-threatening form of the disease. Analysis of COVID-19 in the UK solid organ transplant recipient and waiting list candidate populations, including lung transplant, has been reported. ${ }^{16}$ This evidence, along with our evaluation of practice, will aid decision-making when balancing the risks of continuing transplantation during a viral pandemic. Our analysis identified just 2 lung waiting list candidates with confirmed COVID-19 infection during the study period. The accuracy of COVID-19 data is dependent on additional reporting of results, by centers, to UKTR and could potentially be incomplete. This small number may also, in part, be as a result of strict shielding advice for this patient population, issued on March 21, 2020. ${ }^{17}$

It is important to note that the number of new registrations to the lung transplant waiting list also fell during this period of study. Information on patients who were not yet listed is understandably, not available through analysis of the UKTR. This patient population, whose referral for transplantation may have been delayed or halted, as a result of our response to the pandemic, may have been significantly adversely affected.

The SARS-CoV-2 pandemic has resulted in changes to clinical practice in lung transplantation in the UK. UK lung transplant, and individual centers, have successfully adapted their programs to overcome the challenges of staff sickness, redeployment and potential resource limitations associated with the SARS-CoV-2

\begin{tabular}{|c|c|c|c|c|c|c|c|c|}
\hline \multirow{2}{*}{$\begin{array}{l}\text { Outcome on the UK lung } \\
\text { transplant waiting list } \\
\text { March }\end{array}$} & \multicolumn{2}{|c|}{$\begin{array}{l}\text { Total number } \\
\text { active on } \\
\text { transplant } \\
\text { waiting list } 2020 \\
\text { (19) }\end{array}$} & \multicolumn{2}{|c|}{$\begin{array}{l}\text { Died } 2020 \\
\text { (19) }\end{array}$} & \multicolumn{2}{|c|}{$\begin{array}{l}\text { Removed } \\
2020 \text { (19) }\end{array}$} & \multicolumn{2}{|c|}{$\begin{array}{l}\text { Transplanted } \\
2020 \text { (19) }\end{array}$} \\
\hline & 352 & (337) & 9 & (2) & 3 & (8) & 7 & (13) \\
\hline April & 350 & (332) & 8 & (5) & 1 & (3) & 1 & (21) \\
\hline May & 341 & (325) & 4 & (5) & 1 & (3) & 4 & (19) \\
\hline Total & & & 21 & (12) & 5 & (14) & 12 & (53) \\
\hline
\end{tabular}

TABLE 6 Outcome for patients on the active lung transplant waiting list during the early pandemic period (March to May 2020) compared with 2019. 
pandemic. Changes to clinical practice, including increasing use of technologies to aid follow-up care and reduce the number of patient hospital attendances, have been noted. Staff have reported some positive effects from these changes, including reduced travel and hospital attendance for patients and some improvements to communication, through the use of online platforms. Safe access to hospital services for transplant recipients must be maintained, but development of technologies, including provision of "at-home" monitoring and communication, provides an area for further development and quality improvement in practice. Funding for this innovation, and ensuring equity of access for the patient population, will be required, and understanding the impact of such changes, and the pandemic more widely, on the psychological well-being of the lung transplant patient population provides a potential area for future study.

Hopes that the pandemic would peak and resolve after spring 2020 in the UK are fading. It is increasingly evident that this pandemic will move in waves and, at the time of writing, the volume of cases is once again rising. Anecdotally, center-level decisions surrounding lung transplant activity, are once again being made. As experience in the management of the most severely affected COVID-19 patients grows, it is hoped that the impact of case volume on hospital resource will be less acutely felt than earlier in the year. However, the risks specific to lung transplant candidates, recipients, and the potential lung donor population will not disappear. As evidenced here, continuing activity is vital for the candidate population. To facilitate this, the safety of lung donors must be maintained, with further analysis and reporting of COVID-19 infection in early post-transplant recipients, and continued application of evidenced-based donor and recipient COVID-19 testing protocols. Once approved, a COVID-19 vaccination program for transplant candidates would be invaluable in reducing risk and allowing patients to remain active on the waiting list. Protection of transplant resource capacity is required, with attempts to maintain COVID-light hospitals in line with recommendations for elective surgery practice in the UK during COVID-19.15 This will again, be facilitated by advances in, and implementation of, effective and efficient testing programs. Where geographical spread of COVID-19 is heterogenous, with some lung transplant centers less significantly impacted, transfer of candidates across regions may be considered, maintaining access for all. Reviewing our practice, and sharing experience from this early phase of the pandemic, is fundamental in ensuring the safe continuation of lung transplantation.

\section{CONFLICT OF INTEREST}

There are no conflicts of interest

\section{AUTHOR CONTRIBUTIONS}

Gillian Hardman contributed to concept/design, data analysis, data interpretation, and drafting article. Ruth Sutcliffe contributed to concept/design, data collection, and approval of article. Rachel Hogg and Lisa Mumford contributed to data analysis and statistics. Laura Grocott, Sarah-Jane Mead-Regan, Jane Nuttall, Stephanie Dunn, Richard Quigley, and Philip Seeley contributed to data collection and approval of article. Stephen Clark contributed to approval of article. Nawwar Al-Attar, Karen Booth, John H Dark, John H Dark, and Andrew J Fisher contributed to design, data interpretation, and critical revision of article.

\section{DATA AVAILABILITY STATEMENT}

The data that support the findings of this study are available from the corresponding author upon reasonable request.

\section{ORCID}

Gillian Hardman (D) https://orcid.org/0000-0001-9600-4097

\section{REFERENCES}

1. UK Government. Coronavirus (COVID-19) in th UK; 2020. https:// coronavirus.data.gov.uk/cases. Accessed May 5, 2020.

2. UK Government. Coronavirus (COVID-19): 23 March 20202020. https://www.gov.uk/government/speeches/pm-address-to-thenation-on-coronavirus-23-march-2020. Accessed May 10, 2020.

3. Domínguez-Gil B, Coll E, Fernández-Ruiz M, et al. COVID-19 in Spain: Transplantation in the midst of the pandemic. Am J Transplant. 2020;20(9):2593-2598.

4. Loupy A, Aubert O, Reese PP, Bastien O, Bayer F, Jacquelinet C. Organ procurement and transplantation during the COVID-19 pandemic. Lancet. 2020;395(10237):e95-e96.

5. Angelico R, Trapani S, Manzia TM, Lombardini L, Tisone G, Cardillo M. The COVID-19 outbreak in Italy: Initial implications for organ transplantation programs. Am J Transplant. 2020;20(7):1780-1784.

6. de Vries APJ, Alwayn IPJ, Hoek RAS, et al. Immediate impact of COVID-19 on transplant activity in the Netherlands. Transpl Immunol. 2020;61:101304

7. Felldin M, Søfteland JM, Magnusson J, et al. Initial Report From a Swedish High-Volume Transplant Center After The First Wave of The COVID-19 Pandemic. Transplantation. 2021;105(1):108-114

8. Michel S, Witt C, Gottlieb J, Aigner C. Impact of COVID-19 on Lung Transplant Activity in Germany-A Cross-Sectional Survey. Thorac Cardiovasc Surg. 2021;69(1):92-94.

9. Picard C, Le Pavec J, Tissot A. Impact of the Covid-19 pandemic and lung transplantation program in France. Respir Med Res. 2020;78:100758.

10. NHS Improvement. Coronavirus Specialty guides - management of Cardiothoracic surgery patients 2020. https://www.england.nhs. uk/coronavirus/wp-content/uploads/sites/52/2020/03/specialtyguide-cardiothoracic-surgery-v1-20-march-2020.pdf. Accessed May 5, 2020.

11. NHSBT ODT Clinical. Annual Activity Report 2018/19. Organ specific reports - Cardiothoracic 2019. https://nhsbtdbe.blob.core. windows.net/umbraco-assets-corp/16416/section-7-cardiothor acic-activity.pdf. Accessed November 15, 2020.

12. NHSBT ODT Cliinical. POL304/2 - SARS-CoV-2 Assessment and screening in Organ Donors and Recipients 2020. https://nhsbtdbe. blob.core.windows.net/umbraco-assets-corp/20342/pol304.pdf Accessed October 30, 2020.

13. Ceulemans LJ, Van Slambrouck J, Leyn P, et al. Successful doublelung transplantation from a donor previously infected with SARSCoV-2. Lancet Respir Med. 2021;9(3):315-318.

14. Pasrija C, Shah A, Kaczorowski DJ, Lau CL. Cardiac and Pulmonary Transplant Considerations During COVID-19 Pandemic. Innovations (Phila). 2020;15(4):314-316.

15. England RCoSo. Elective surgery during COVID-19 2020. https:// www.rcseng.ac.uk/news-and-events/news/archive/survey-resul ts-elective-surgery-under-covid/. Accessed December 10, 2020. 
16. Ravanan R, Callaghan CJ, Mumford L, et al. SARS-CoV-2 infection and early mortality of wait-listed and solid organ transplant recipients in England: a national cohort study. Am J Transplant. 2020;20(11):3008-3018.

17. UK Government. Guidance on shielding and protecting extremely vulnerable persons from COVID-19 2020. https://www.gov.uk/ government/publications/guidance-on-shielding-and-protecting -extremely-vulnerable-persons-from-covid-19. Accessed November 20, 2020.
How to cite this article: Hardman G, Sutcliffe R, Hogg R, et al; the NHS Blood, Transplant Cardiothoracic Advisory Group Clinical Audit Group. The impact of the SARS-CoV-2 pandemic and COVID-19 on lung transplantation in the UK: Lessons learned from the first wave. Clin Transplant. 2021;35:e14210. https://doi.org/10.1111/ctr.14210 